\title{
SAR image reconstruction by expectation maximization based matching pursuit
}

\author{
S. Uğur ${ }^{\mathrm{a}, *}$, O. Arıkan ${ }^{\mathrm{b}}$, A. Cafer Gürbüz ${ }^{\mathrm{c}}$ \\ a Meteksan Savunma, Ankara, Turkey \\ ${ }^{\mathrm{b}}$ Bilkent University, Electrical and Electronics Engineering Department, Ankara, Turkey \\ c Department of Electrical and Electronics Engineering, TOBB University of Economics and Technology, Ankara, Turkey
}

\section{A R T I C L E I N F O}

\section{Article history:}

Available online 10 November 2014

\section{Keywords:}

Synthetic Aperture Radar

Expectation Maximization based Matching

Pursuit algorithm

Compressed sensing

Autofocus

\begin{abstract}
A B S T R A C T
Synthetic Aperture Radar (SAR) provides high resolution images of terrain and target reflectivity. SAR systems are indispensable in many remote sensing applications. Phase errors due to uncompensated platform motion degrade resolution in reconstructed images. A multitude of autofocusing techniques has been proposed to estimate and correct phase errors in SAR images. Some autofocus techniques work as a post-processor on reconstructed images and some are integrated into the image reconstruction algorithms. Compressed Sensing (CS), as a relatively new theory, can be applied to sparse SAR image reconstruction especially in detection of strong targets. Autofocus can also be integrated into CS based SAR image reconstruction techniques. However, due to their high computational complexity, CS based techniques are not commonly used in practice. To improve efficiency of image reconstruction we propose a novel CS based SAR imaging technique which utilizes recently proposed Expectation Maximization based Matching Pursuit (EMMP) algorithm. EMMP algorithm is greedy and computationally less complex enabling fast SAR image reconstructions. The proposed EMMP based SAR image reconstruction technique also performs autofocus and image reconstruction simultaneously. Based on a variety of metrics, performance of the proposed EMMP based SAR image reconstruction technique is investigated. The obtained results show that the proposed technique provides high resolution images of sparse target scenes while performing highly accurate motion compensation.
\end{abstract}

(C) 2014 Elsevier Inc. All rights reserved.

\section{Introduction}

Synthetic Aperture Radar (SAR) is a technique to generate high resolution images of ground reflectivity from a sensor platform. Over more than five decades of their use, SAR systems have found wide variety of application areas ranging from military surveillance to environmental monitoring activities. The success of SAR systems stems from their ability to coherently integrate multiple returns acquired over the course of the flight path of the SAR platforms, which requires precise platform position information within a fraction of the carrier wavelength. Even with the use of modern navigational systems, there is an error due to the difference between actual and the estimated platform positions which results in considerable phase errors especially for high resolution SAR systems typically operating at higher carrier frequencies. Several autofocus techniques have been developed to estimate this residual phase

\footnotetext{
* Corresponding author.

E-mail addresses: sugur@meteksan.com (S. Uḡur), oarikan@ee.bilkent.edu.tr (O. Arıkan), acgurbuz@etu.edu.tr (A.C. Gürbüz).
}

error [1-7]. Once a reliable estimate is obtained, the effect of the phase error is compensated on the raw SAR data to provide better SAR reconstructions.

Compressed Sensing (CS) is a relatively new paradigm $[8,9]$ in which theoretically, sparse signals can be reconstructed by sampling them below Nyquist rate. Application of CS requires the reconstructed signal to be sparse in a known basis [10]. Since sparsity is encountered in many natural signals, CS has found diverse application areas including radar signal processing [11-18]. Compressive sensing based radar in theory has several advantages such as reduced memory size, decreased A/D converter rates or possibility of eliminating the match filtering process [19]. Because CS allows to reconstruct SAR images by using data sampled below the Nyquist rate, the required memory size and A/D converter rate can be relaxed, resulting important cost and complexity savings in practice [20].

In the application of CS to SAR image reconstruction, the scene reflectivity is required to have a sparse representation in a known basis. Speckle noise creates significant challenges in representation of SAR images sparsely. But for radar scenes with highly reflective man-made objects, wavelets [21], standard unit impulse basis 
vectors, or both can be used for a sparse representation of the targets that dominate the scene reflectivity.

SAR image reconstruction by using sparsity driven penalty function has been investigated in [19,22-25]. Ref. [26] gives a thorough survey of recent literature on sparsity driven SAR imaging. CS based SAR imaging is generally formulated as a convex $l_{1}$ norm minimization problem and it is solved by either linear programming or greedy pursuit algorithms. Although these techniques do not consider phase errors in SAR image reconstruction, the proposed techniques in [20,27-29] provide sparse reconstructions in the presence of phase errors. However, compared to the commonly used SAR autofocusing techniques, these approaches require significantly more processing time than conventional reconstruction techniques that limits their practical use.

In the present study, a novel SAR reconstruction technique that utilizes a new sparse reconstruction approach called as Expectation Maximization Matching Pursuit (EMMP) algorithm [30] is proposed. The EMMP algorithm uses the compressive measurements as incomplete data about the system and iteratively applies expectation and maximization (EM) steps to construct the complete data that would correspond to a set of SAR data for each dominant target in the scene. The objective of EM iterations is to provide more reliable estimates to the complete data so that accurate and efficient estimation of the individual target parameters can be obtained more reliably in the maximization step. Once, more accurate estimates for a certain target are obtained, its contribution to the incomplete data can be more accurately estimated allowing reconstruction of remaining targets without its interference. This EM procedure also allows to estimate unknown phases for each complete data component in an iterative manner.

The proposed EMMP based SAR imaging algorithm is greedy, computationally less complex, and has lower reconstruction errors compared to $l_{1}$ norm minimization. Hence, both the accuracy and convergence rate of the iterations significantly increase, enabling fast and high resolution SAR image reconstructions. Note that, in addition to the preliminary results presented in [31], the proposed approach [32] is extended to conduct autofocus as part of the EMMP iterations. As illustrated on both synthetic and real data sets, the proposed EMMP based SAR reconstruction technique performs highly effective autofocus in the presence of phase errors.

In Section 2, the proposed technique of simultaneous reconstruction and autofocus of sparse SAR images based on EMMP algorithm is described. Section 3 investigates the effect of sparsity parameter on the image reconstruction quality of the proposed technique. Comparison of the image reconstruction performances of the proposed technique and the technique based on the nonlinear conjugate gradient descent algorithm is given in Section 4. Section 5 concludes the article.

\section{Simultaneous reconstruction and autofocus of sparse SAR images based on EMMP algorithm}

In spotlight mode SAR, an airborne or spaceborne platform carries a mono-static radar system on a straight flight path, while the radar transmits and receives echoes from the area of interest (see Fig. 1). The received and digitized radar returns are coherently processed to obtain significantly higher resolutions in azimuth direction that could have been obtained by a large aperture antenna. Baseband measurement model of a spotlight SAR system can be written as the following vector-matrix equation [25]:

$\mathbf{y}=\mathbf{G} \mathbf{x}+\mathbf{w}$,

where $\mathbf{y}$ is the received signal (the measurement vector), $\mathbf{G}$ is the complex valued discrete SAR projection operator matrix, $\mathbf{x}$ is the reflectivity vector and $\mathbf{w}$ is the additive complex white Gaussian

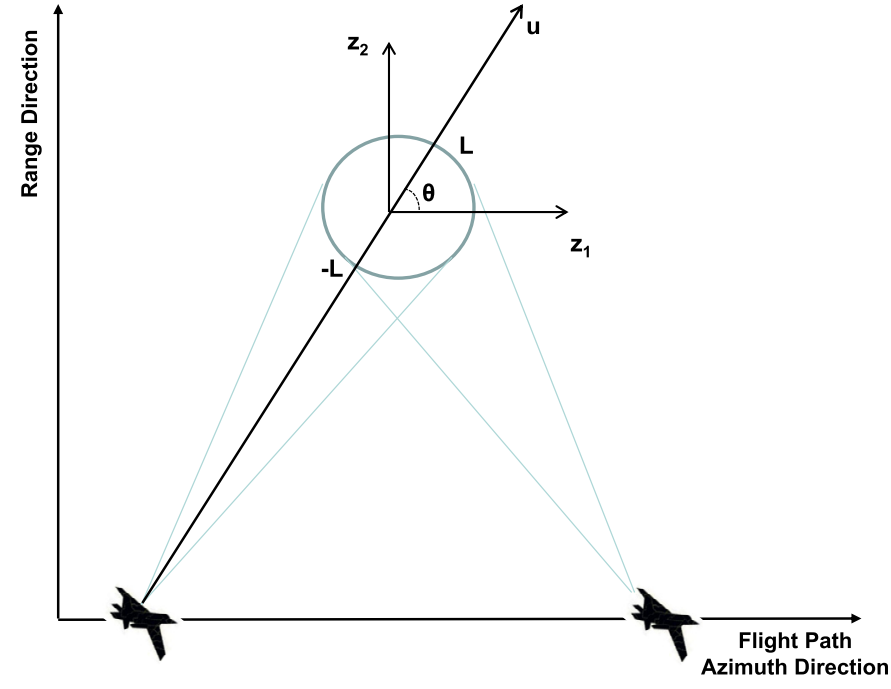

Fig. 1. Spotlight mode SAR imaging geometry.

measurement noise vector. Assuming that the reconstruction will be performed over a target grid of $N \times N$ range and azimuth samples, then, $\mathbf{y}, \mathbf{x}$, and $\mathbf{w}$ are $m \times 1$ vectors and $\mathbf{G}$ is a matrix of size $m \times m$, respectively, for $m=N^{2}$.

One important application of SAR systems is imaging of manmade objects. Since, typical reflections from man-made objects are significantly stronger than that of background terrain, reflectivity distribution over the imaged area can be modeled as a sparse distribution over an appropriate set of vectors such as wavelets.

Proven guarantees of CS based reconstruction techniques ensure that reliable reconstruction of a sparse signal of length $m$ is possible if the measurement matrix satisfies Restricted Isometry Property (RIP) and the number of measurements are at least $O(K \log (m / K))$ where $K$ is the level of sparsity of the signal [33], which can be significantly smaller than $m$. Thus, for sparse SAR image reconstructions, the required number of samples can be significantly lower than the Nyquist rate, providing important hardware savings. To exploit the potential reduction in the sampling rate, the method described in [20] can be used to under-sample the measured data. Assuming that the reflectivity vector is sparse in the column space of a given matrix $\boldsymbol{\Psi}$ with representation coefficients $\boldsymbol{\alpha}$, measurement model given in (1) can be written equivalently as:

$\mathbf{y}=\mathbf{G} \Psi \boldsymbol{\alpha}+\mathbf{w}=\mathbf{A} \boldsymbol{\alpha}+\mathbf{w}$.

In CS applications, it is desired to obtain a reconstruction which is as sparse as possible while providing a tolerable fit to measurements. For this purpose, $l_{0}$ norm of $\boldsymbol{\alpha}$ can be minimized $[8,9]$. Since $l_{0}$ norm optimization requires combinatoric search that is rarely feasible in practice, generally the $l_{0}$ norm problem is relaxed to $l_{1}$ norm minimization problem. It is proven that $l_{0}$ and $l_{1}$ norm minimization problems provide the same solution if $\boldsymbol{\alpha}$ is sparse and $\mathbf{A}$ holds the RIP $[34,35]$.

Generally, the SAR image reconstruction in CS methodology has been formulated in two different approaches. In Basis Pursuit Denoising (BPDN) [36] formulation,

$\min _{\boldsymbol{\alpha}}\|\boldsymbol{\alpha}\|_{1}$ such that $\|\mathbf{y}-\mathbf{A} \boldsymbol{\alpha}\|_{2} \leq \sigma$,

the scene with minimum $l_{1}$ norm is reconstructed such that the resulting fit error to measurements is less than a threshold $\sigma$. In LASSO formulation [37],

$\min _{\boldsymbol{\alpha}}\|\mathbf{y}-\mathbf{A} \boldsymbol{\alpha}\|_{2} \quad$ such that $\quad\|\boldsymbol{\alpha}\|_{1} \leq \tau$, 
the scene whose reflectivity has an $l_{1}$ norm that is less than $\tau$, is chosen to minimize the fit error. Although in principle these formulations are equivalent for a properly chosen $(\sigma, \tau)$ pair, it is not straightforward to determine $\sigma$ for SAR image reconstructions especially if the terrain reflectivity is highly variable. However, an appropriate choice for $\tau$ can be obtained based on the size and reflectivity of the dominant reflectors in the imaged area. Hence, it is easier to choose a proper $\tau$, to the $l_{1}$-norm of the target. Therefore in practice, LASSO formulation is more suitable for SAR image reconstructions with dominant reflectors in the target scene.

Unlike BPDN and LASSO formulations, the proposed EMMP approach provides a near optimal solution to the following $l_{0}$ norm problem:

$\min _{\boldsymbol{\alpha}}\|\mathbf{y}-\mathbf{A} \boldsymbol{\alpha}\|_{2} \quad$ such that $\quad\|\boldsymbol{\alpha}\|_{0} \leq K$,

where $K$ is the sparsity level of the signal. Sparsity level $K$ can be estimated for man-made targets based on the ratio of the target and resolution cell areas. Hence, it is actually easier to choose $K$, because the choice for $\tau$ in the LASSO formulation also requires reflectivity information about these targets.

SAR systems need accurate distance and angle information between the SAR platform and the reference point in the terrain of interest in order to establish the synthetic aperture precisely. However, especially in airborne SAR applications, due to the limited accuracy of the navigational sensors, there is always some residual error left in the estimation of the actual flight path. These uncompensated platform motion errors cause uncertainties in distance and angle measurements which result in phase errors in the received SAR signal. Let $\phi(t)$ represents the phase error which results mixing errors, hence the demodulation output of the SAR system becomes [38]:

$y_{p}(t)=e^{j \phi(t)} y(t)$

where $y_{p}(t)$ is the received signal with phase error due to an error of the platform position from the scene center. Following (6), the exponential multiplication of the phase error can be inserted to the signal model and the measurement relation of (1) becomes:

$\mathbf{y}_{p}=\boldsymbol{\Phi} \mathbf{y}=\boldsymbol{\Phi} \mathbf{G} \mathbf{x}+\mathbf{w}$.

Here, $\boldsymbol{\Phi}$ is a diagonal matrix representing phase errors for every different measurement position on the flight path and is given as:

$\boldsymbol{\Phi}=\left(\begin{array}{cccc}e^{j \phi_{1}} & & & \\ & e^{j \phi_{2}} & & \\ & & \ddots & \\ & & & e^{j \phi_{N \times N}}\end{array}\right)$.

Here $N \times N$ is the total number of measurement positions that will be used in the image reconstruction and $\phi_{i}$ 's are the corresponding phase errors in radians incurred at the $i$ th measurement position. Because the deviation from the flight path is typically a small fraction of a range resolution bin, the error in the range compressed data due to phase error is generally ignored [38]. Therefore, phase errors are assumed to be the same for all the obtained data at each azimuth position of the radar platform, resulting:

$$
\left(\begin{array}{c}
y_{p_{1}} \\
y_{p_{2}} \\
\vdots \\
y_{p_{N}}
\end{array}\right)=\left(\begin{array}{llll}
e^{j \phi_{1}} & & & \\
& e^{j \phi_{2}} & & \\
& & \ddots & \\
& & & e^{j \phi_{N}}
\end{array}\right)\left(\begin{array}{c}
G_{1} \\
G_{2} \\
\vdots \\
G_{N}
\end{array}\right) \mathbf{x}+\mathbf{w},
$$

where $y_{p_{i}}$ is the partition of the measurement vector $\mathbf{y}_{p}$ which contains all the range points corresponding to the azimuth position index $i, \phi_{i}$ is the respective phase error, $G_{i}$ is the partition of the matrix $G$ for each azimuth position and $N$ is the total number of azimuth positions.

To obtain a sparse and autofocused reconstruction, we would like to solve the following optimization in terms of two sets of variables, $\boldsymbol{\alpha}$ and $\boldsymbol{\Phi}$ to account for the phase error:

$\min _{\boldsymbol{\alpha}, \boldsymbol{\Phi}}\left\|\mathbf{y}_{p}-\boldsymbol{\Phi} \mathbf{A} \boldsymbol{\alpha}\right\|_{2} \quad$ such that $\quad\|\boldsymbol{\alpha}\|_{0} \leq K$.

The required optimization in (10) can be reduced to an optimization over $\boldsymbol{\alpha}$ alone by replacing $\boldsymbol{\Phi}$ that provides the minimum cost for each $\boldsymbol{\alpha}$ [39]:

$\min _{\boldsymbol{\alpha}} f_{0}(\boldsymbol{\alpha})$ such that $\|\boldsymbol{\alpha}\|_{0} \leq K$,

where,

$f_{0}(\boldsymbol{\alpha})=\inf _{\boldsymbol{\Phi}}\left\{\left\|\mathbf{y}_{p}-\boldsymbol{\Phi} \mathbf{A} \boldsymbol{\alpha}\right\|_{2}\right\}$.

To minimize the cost in (12) for a given $\boldsymbol{\alpha}$, the phase error matrix $\boldsymbol{\Phi}$ that minimizes $\left\|\mathbf{y}_{p}-\boldsymbol{\Phi} \mathbf{A} \boldsymbol{\alpha}\right\|_{2}$ should be obtained. Because phase errors are assumed to be the same for all the data corresponding to a certain azimuth location [38], the minimization can be formulated equivalently as:

$\underset{\boldsymbol{\Phi}}{\inf }\left\|\mathbf{y}_{p}-\boldsymbol{\Phi} \mathbf{A} \boldsymbol{\alpha}\right\|_{2}=\sum_{i} \underset{\phi_{i}}{\inf }\left\|\mathbf{y}_{p_{i}}-e^{j \phi_{i}} \mathbf{A}_{i} \boldsymbol{\alpha}\right\|_{2}$

where $\mathbf{A}_{i}$ is the partition of the matrix $\mathbf{A}$ corresponding to the $i$ th azimuth position. In this formulation, the unique solution for phase error estimate $\phi_{i}$, for each azimuth position can be obtained as:

$\hat{\phi}_{i}=\angle\left(\boldsymbol{\alpha}^{H} \mathbf{A}_{i}^{H} \mathbf{y}_{p_{i}}\right)$.

With this result, (10) can be reduced to an optimization over $\boldsymbol{\alpha}$ only:

$$
\begin{array}{ll}
\min _{\boldsymbol{\alpha}}\left\|\mathbf{y}_{p}-\boldsymbol{\Phi} \mathbf{A} \boldsymbol{\alpha}\right\|_{2} \quad \text { such that } & \|\boldsymbol{\alpha}\|_{0} \leq K, \\
& \phi_{i}=\angle\left(\boldsymbol{\alpha}^{H} \mathbf{A}_{i}^{H} \mathbf{y}_{p_{i}}\right) .
\end{array}
$$

To obtain both the phase error estimates and autofocused image parameters (14) and (15) are sequentially used. EMMP iterations are used for (15) and phase error corrections are easily implemented within these iterations as summarized in Table 1. In the algorithm, $\mathbf{C}$ corresponds to the complete data matrix, $\mathbf{r}$ corresponds to the residual vector and $\epsilon$ corresponds to the termination criteria which can be set to the average energy of background pixels around the region of interest. The inputs of the EMMP algorithm are the measurement matrix $\mathbf{A}$, the measurements $\mathbf{y}_{p}$, the sparsity level $K$, and the termination parameter $\epsilon$. Initially, the complete data matrix is set to zero and the residual vector is initialized to $\mathbf{y}_{p}$. Because EM algorithm is known to be locally convergent, its initialization plays a critical role in ensuring convergence to the global solution. But with the initial values set as indicated, the EMMP algorithm always gives meaningful results. Whether these solutions correspond to the global solution or, they are local solutions in the vicinity of the global one giving acceptable results, shall be investigated as a further work.

As in the EM algorithm [40], in EMMP algorithm iterations, given estimates for $\mathbf{y}_{j}$ and $\boldsymbol{\Phi}$, the $j$ th sparse component of $\boldsymbol{\alpha}$ is found as the best matching vector among the columns of $\mathbf{A}$ to $\boldsymbol{\Phi}^{-1} \mathbf{y}_{j}$, for $1 \leq j \leq K$. The selected index list and the complete data matrix are updated. Then, $\boldsymbol{\Phi}$ is re-estimated by using the obtained $\boldsymbol{\alpha}$, and the iterations are restarted. The iterations are continued until the termination criteria or a pre-determined number of iterations are reached. 
Table 1

EMMP algorithm with phase error estimation.
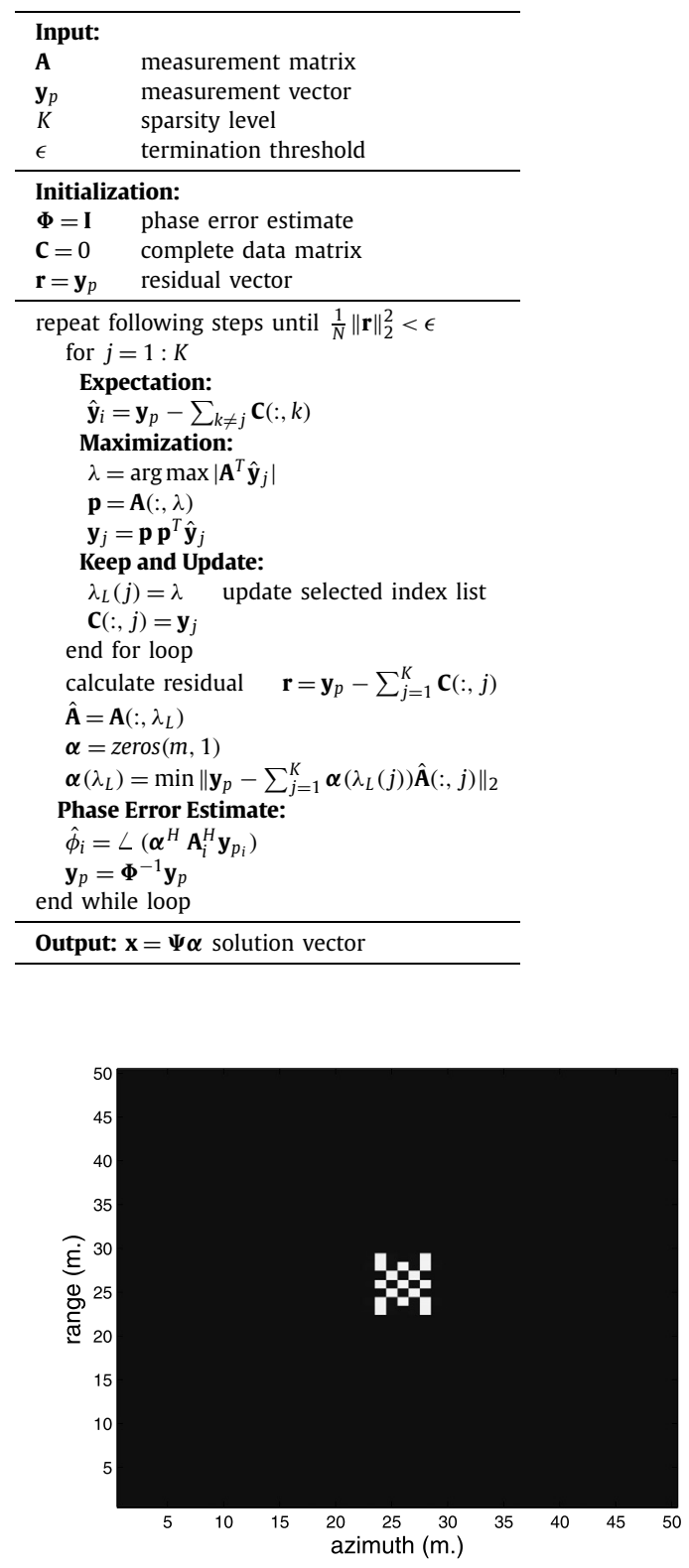

(a)

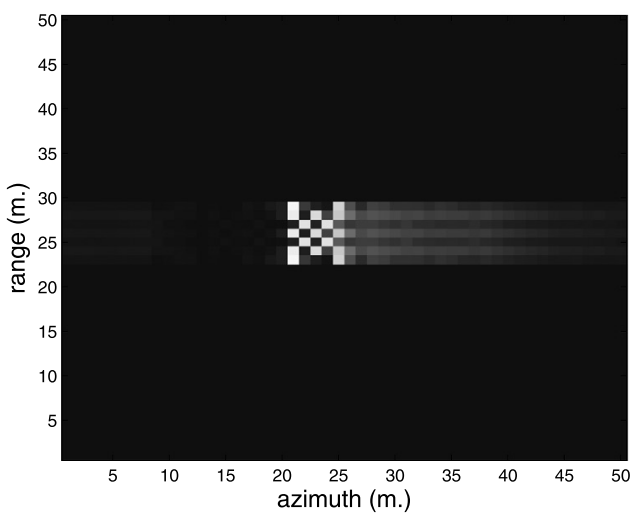

(c)

\subsection{Simulation results}

To illustrate the performance of the proposed EMMP based autofocused SAR imaging technique (EMMP-AF-SAR) reconstructions on both synthetic data as well as real SAR data from MSTAR database [41] have been investigated. For synthetic data, an A/D converter operating at one-third of the Nyquist rate is used. For Slicy data of MSTAR database, an A/D converter operating at onefourth of the Nyquist rate is used. In addition to the rate reduction of the A/D converter, another $10 \%$ reduction on the obtained samples is achieved by using pseudo-random sampling scheme detailed in [20].

MATLAB implementation of the proposed EMMP-AF-SAR technique running on a standard laptop converges in about 20 iterations taking 20 seconds to 5 minutes depending on the size of the image, which is significantly faster than alternative gradient descent based optimization techniques for CS reconstructions. For real-time applications, the proposed approach can be implemented on off-the-shelf processor boards to further reduce the computation time.

\subsubsection{Results on synthetic data}

The proposed EMMP-AF-SAR technique is first applied on a set of synthetic SAR data. To provide a benchmark, the synthetic SAR data with no phase error is processed with Polar Format Algorithm (PFA) and the resultant image shown in Fig. 2(a) is obtained. To investigate the effect of motion errors, the synthetic SAR data is distorted by phase errors at each azimuth vantage point. The result obtained by the PFA algorithm is shown in Fig. 2(b). For comparison, reconstructed SAR image by using the well known Phase Gra-

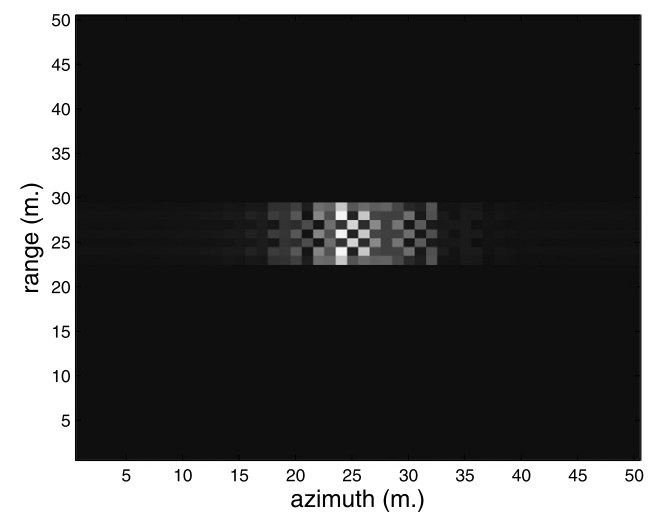

(b)

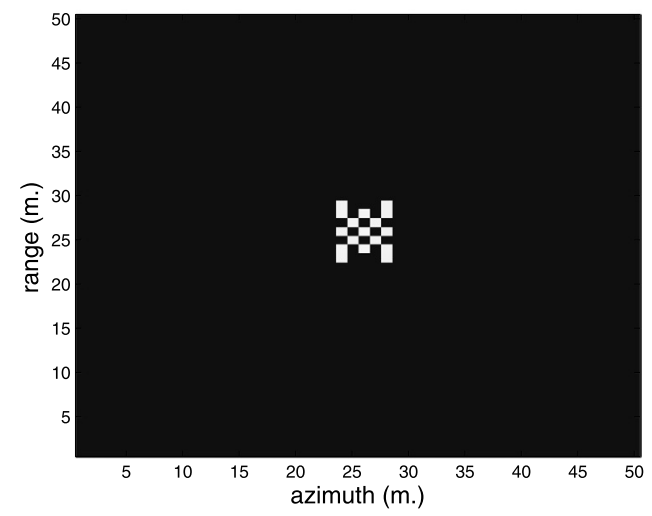

(d)

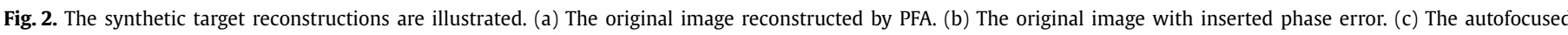

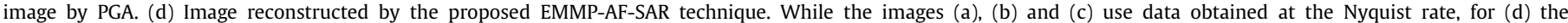
EMMP-AF-SAR technique uses only $30 \%$ of the Nyquist rate data. 
(1)
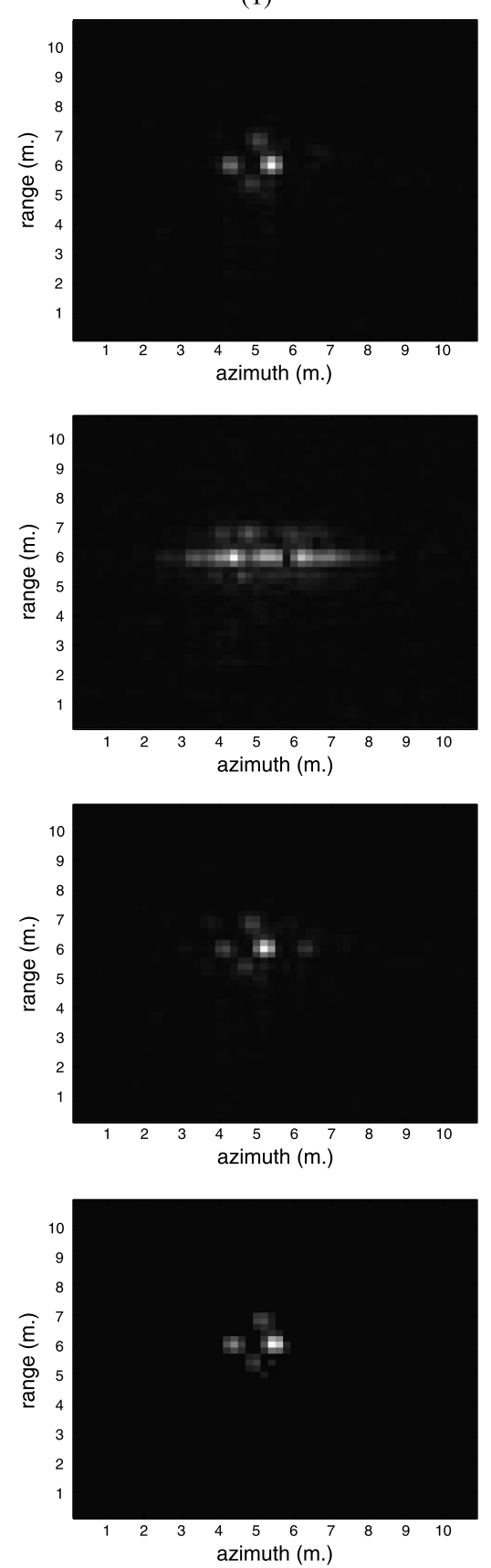

(2)
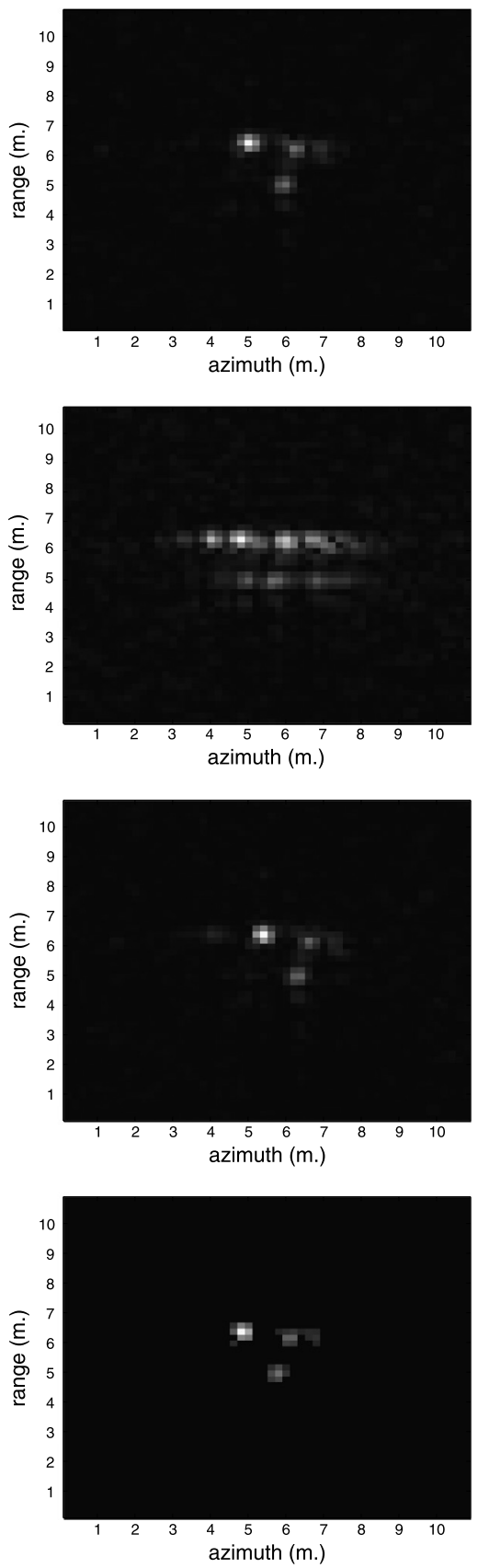

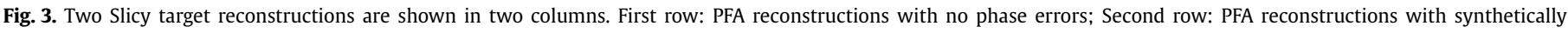

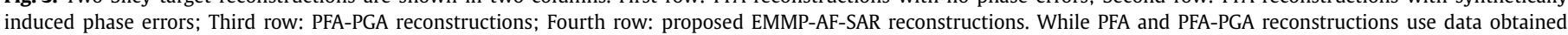
at the Nyquist rate, the EMMP-AF-SAR technique uses only $22.5 \%$ of the Nyquist rate data.

dient Autofocus algorithm (PGA) [7] is shown in Fig. 2(c). Usually, three iterations of PGA are sufficient to compensate phase errors reasonably. As seen from Fig. 2(c), although the image is corrected to some extent compared to Fig. 2(b), phase error related degradations are still visible. In Fig. 2(d), the reconstruction obtained by using the proposed EMMP-AF-SAR technique is shown. Although this reconstruction is obtained by using only $30 \%$ of the data required by the PGA technique, it is visibly better focused than the result of PGA shown in Fig. 2(c).

\subsubsection{Results on real Slicy SAR data}

The results obtained using real SAR measurements for two types of Slicy targets are shown in Fig. 3. The first row presents PFA reconstructions with no phase error. The PFA reconstructions with synthetically induced phase error are shown in the second row. Reconstructed by PFA and autofocused by PGA images are given in the third row. The fourth row presents the reconstructions obtained by the proposed EMMP-AF-SAR technique. Note that in the reconstructions with the proposed EMMP-AF-SAR technique only $22.5 \%$ of the raw data is used.

The results show that the reconstruction quality of the proposed EMMP-AF-SAR technique is better than that of the PFA-PGA technique while the proposed EMMP-AF-SAR technique requires a fraction of the data for reconstruction. Even though some minor blurs caused by phase errors left in PFA-PGA reconstructions, phase error degradations are almost totally removed from the images reconstructed by the proposed EMMP-AF-SAR technique. Unlike the previously proposed CS SAR reconstruction techniques, the 


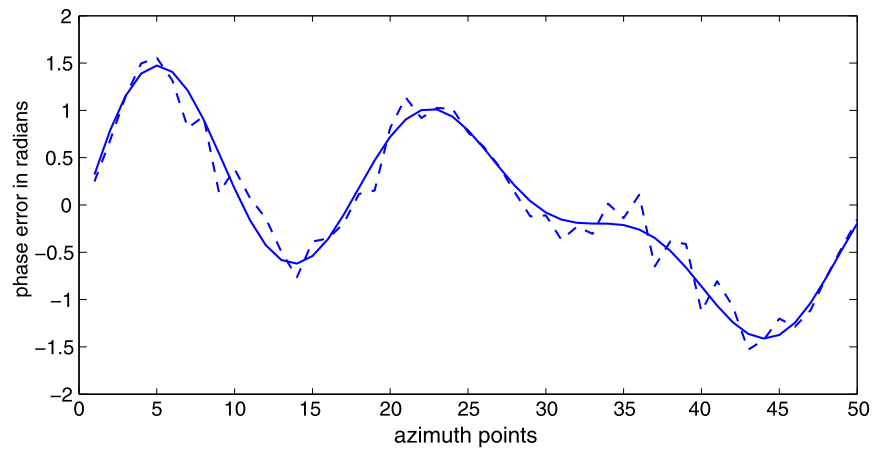

Fig. 4. The synthetically inserted (solid line) and the estimated phase errors (dashed line) in radians.

Table 2

Metrics for the Slicy target imagery reconstructed by PFA-PGA and the proposed EMMP-AF-SAR techniques. The PFA-PGA technique, which is reconstructed by PFA and autofocused by PGA uses whole raw SAR data. The proposed EMMP-AF-SAR technique uses only $22.5 \%$ of the Nyquist rate data.

\begin{tabular}{llllll}
\hline & \multicolumn{2}{l}{ Slicy target 1} & & \multicolumn{3}{l}{ Slicy target 2} & \\
\cline { 2 - 3 } \cline { 5 - 6 } & MSE & & & MSE & $H$ \\
\hline PFA-PGA & $4.0 \times 10^{-3}$ & 4.91 & & $9.3 \times 10^{-4}$ & 4.59 \\
EMMP-AF-SAR & $9.1 \times 10^{-3}$ & 0.14 & & $5.8 \times 10^{-5}$ & 0.12 \\
\hline
\end{tabular}

processing time of the proposed EMMP-AF-SAR technique is also comparable to the PFA-PGA technique.

To illustrate the extend of autofocusing provided by the proposed algorithm, significantly large phase error is synthetically induced in the raw data. As shown in Fig. 4, the proposed EMMP-AFSAR technique provides an acceptable estimate to the synthetically induced phase error.

In order to quantify and compare the image reconstruction performance of the EMMP-AF-SAR technique with alternative techniques, the following two metrics are used:

1. Mean Square Error, which is defined as [24]:

$$
M S E=\frac{1}{N^{2}}\||\mathbf{x}|-|\hat{\mathbf{x}}|\|_{2}^{2},
$$

where $\mathbf{x}$ is the original image, $\hat{\mathbf{x}}$ is the reconstructed image, and $N^{2}$ is the total number of pixels in the image.

2. Entropy of the image [24]: this is a metric related to sharpness of the image:

$$
H(\mathbf{x})=-\sum_{i} p_{i} \log _{2} p_{i}
$$

where the discrete variable $p$ contains the histogram counts of the image $\mathbf{x}$. Entropy is small for sharper images so it is preferable for an algorithm to result in low entropies for image formation.

These metrics give indications about the performance of the reconstructions especially on the target classification applications. Table 2 lists these metrics for the images illustrated in Fig. 3. The data presented in Table 2 indicates that PFA-PGA technique has a better performance for the MSE metric of the Slicy 1 target. For other parameters, the EMMP-AF-SAR technique outperforms the PFA-PGA technique. Note that, for the scenes with strong manmade targets, EMMP-AF-SAR technique provides reconstructions with only non-zero values present in the support of the targets, thus, eliminates the speckle noise associated with ground reflectivity. The results show that the EMMP-AF-SAR technique serves well for the target classification without further windowing and speckle noise removal. It is important to note that, unlike the classical PFA-PGA technique, the proposed EMMP-AF-SAR technique has variable $\epsilon$ and $K$ parameters that can be adjusted for the target class of interest to provide significant control over the reconstructions.

\section{Effect of sparsity on quality of SAR image reconstructions}

In this section the effect of the sparsity parameter, $K$, on the reconstructed image quality is investigated. For this investigation, military target image of MSTAR database is used with $40 \%$ of the Nyquist rate data. Fig. 5(a) gives the original image used in the trials which is reconstructed by PFA. The image contains a military target with very high speckle noise. Figs. 5(b)-(f) illustrate the resultant images reconstructed by the proposed EMMP-AF-SAR technique for a range of sparsity level $K$.

Fig. 5 illustrates that for $K$ set lower than the actual sparsity level of the target, reconstructed images lack important features of the target. For $K$ set close to the actual sparsity level of the target (which is approximately 50), reconstructions provide better results. For $K$ set to greater than the actual sparsity of the target, the result is the increased noise level in the reconstructed images. Quantitative metrics given in Table 3 also support these observations. Again the best results are obtained for the reconstructions with $K$ set close to the actual sparsity of the target.

\section{Performance comparison between EMMP-AF-SAR and non-linear conjugate gradient descent algorithms}

In this section, the image reconstruction quality of the proposed technique is compared to that of a previously proposed CS based SAR image reconstruction technique based on gradient descent algorithm that will be referred to as CS-PE-TV [20]. The original MSTAR target images, the synthetic motion error induced images both reconstructed by PFA, and the images reconstructed by PFA and autofocused by PGA are again given in the rows (a), (b), and (c) of Fig. 6 as a benchmark. Row (d) of Fig. 6 gives the images reconstructed by the CS-PE-TV technique. Reconstructions obtained by the EMMP-AF-SAR technique for the same target scenes are shown in row (e) of Fig. 6 . Note that, only $40 \%$ of the Nyquist sampled raw data is used in these CS reconstructions. Compared to the CS-PE-TV reconstructions given in row (d) of Fig. 6, reconstructions provided by the EMMP-AF-SAR technique are of similar quality. Also, it is observed that the EMMP-AF-SAR technique provides an effective autofocusing on the reconstructions. Compared to the CS-PE-TV technique, the main advantage of the EMMP-AF-SAR technique is its computational efficiency. An MSTAR reconstruction with the non-linear conjugate gradient descent algorithm takes about 20 hours while the same reconstruction with the EMMP-AF-SAR technique takes only 3-5 minutes on an ordinary PC. Also, EMMPAF-SAR technique provides reconstructions with significantly more suppressed speckle noise.

While suppressing speckle noise of the terrain, the EMMP-AFSAR technique also removes shadows of targets in the imaged scene. Shadow information in SAR images is valuable and can be used in image classification applications. The technique based on the non-linear conjugate gradient descent algorithm also suppresses shadows. Generally CS based SAR image reconstruction techniques construct only target features but suppress shadows. Retaining shadows in SAR images while reconstructing them by CS techniques is important and will be investigated as a future work. Multichannel autofocus [5,42] and filtered variation [43] are two candidates to preserve the shadow information in CS SAR image reconstruction. 


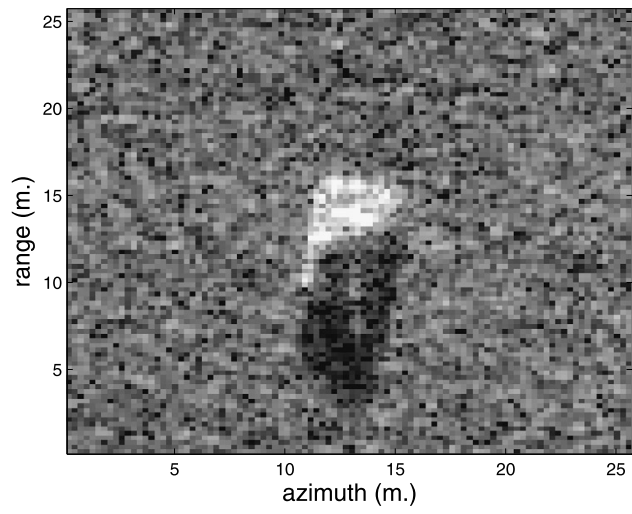

(a)

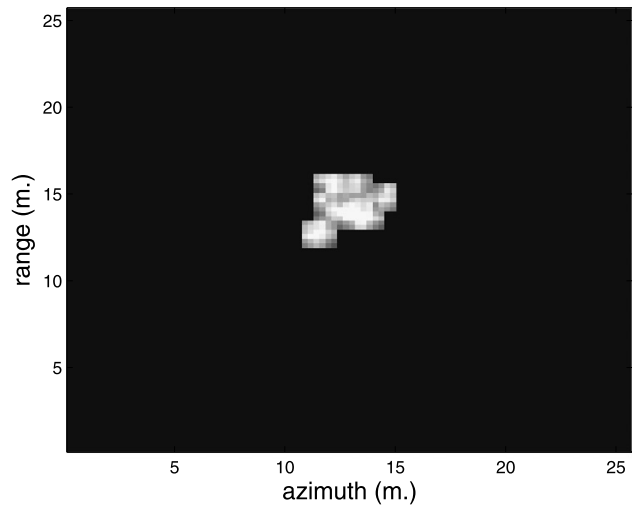

(c)

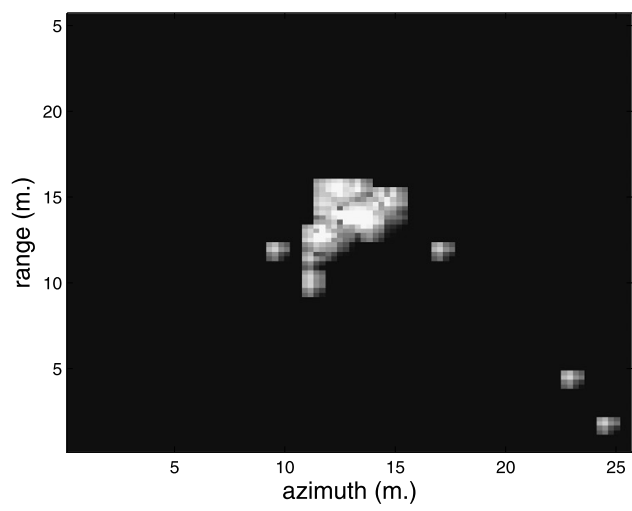

(e)

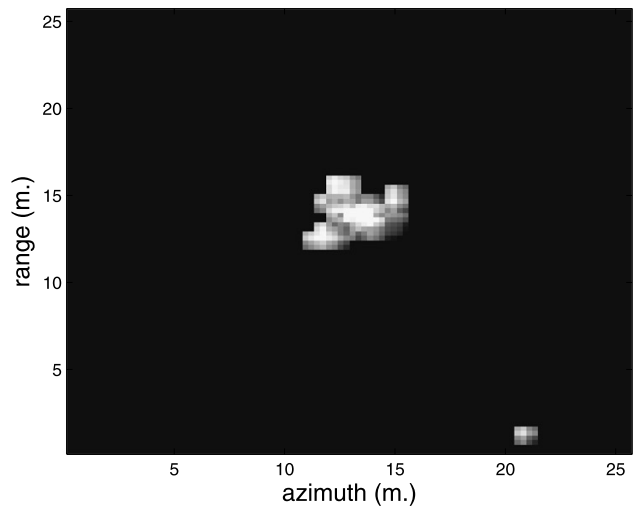

(b)

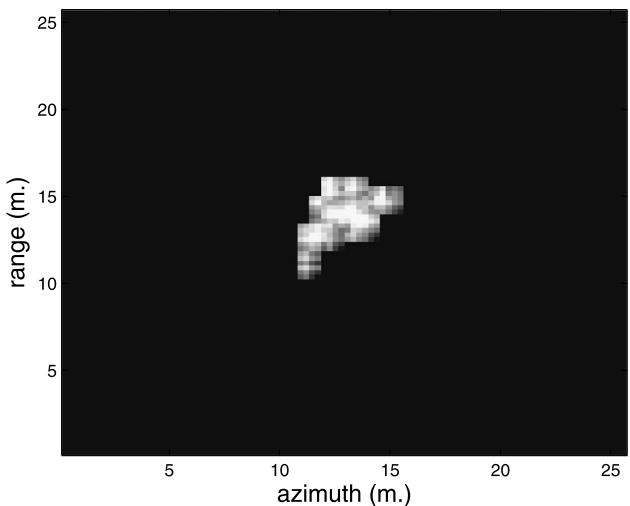

(d)

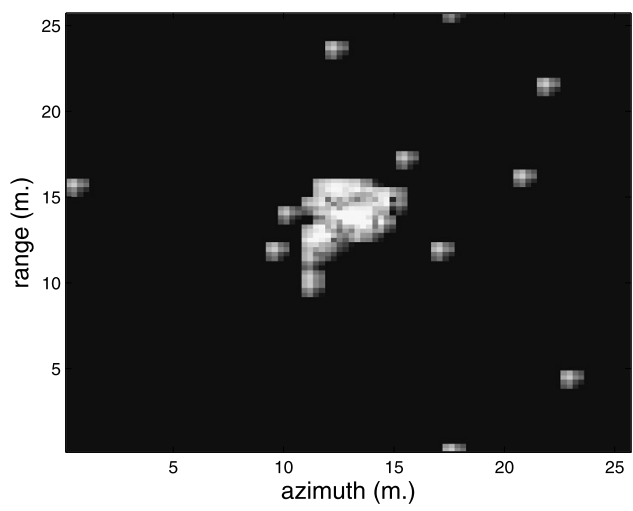

(f)

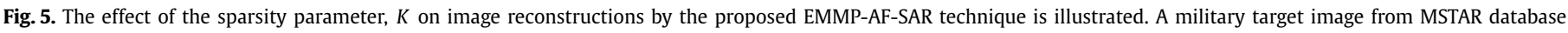

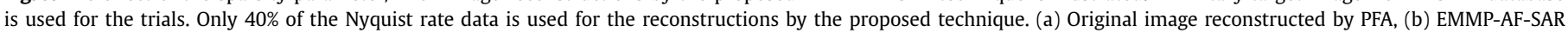
with $K=30$, (c) EMMP-AF-SAR with $K=40$, (d) EMMP-AF-SAR with $K=50$, (e) EMMP-AF-SAR with $K=70$, and (f) EMMP-AF-SAR with $K=80$.

Table 3

Effect of sparsity parameter on image reconstruction quality metrics. A military target image from MSTAR database is used for the trials. Only $40 \%$ of the raw data is used for the reconstructions by the proposed technique.

\begin{tabular}{lll}
\hline$K$ & MSE & $H$ \\
\hline 30 & $5.0 \times 10^{-3}$ & 0.32 \\
40 & $2.7 \times 10^{-3}$ & 0.26 \\
50 & $1.9 \times 10^{-3}$ & 0.32 \\
60 & $2.6 \times 10^{-3}$ & 0.46 \\
70 & $2.5 \times 10^{-3}$ & 0.49 \\
80 & $2.3 \times 10^{-3}$ & 0.60 \\
\hline
\end{tabular}

The performance metrics for MSTAR images reconstructed by different techniques are given in Table 4. For the MSE metric,
EMMP-AF-SAR technique gives a better result than the result of the CS-PE-TV technique, for target (1). But for other two targets, the results of the CS-PE-TV technique are better. In terms of the MSE, it is observed that there is no significant quantitative difference between the reconstructions of these algorithms. However, in terms of Entropy metric, the EMMP-AF-SAR technique provides significantly better reconstructions. Almost total removal of the speckle noise of the terrain provides the edge for the EMMP-AFSAR technique over the CS-PE-TV technique. To achieve improved performance levels with respect to Entropy metric with the reconstructions of the CS-PE-TV technique, a post-processing step designed to suppress the speckle noise can be incorporated to the processing chain. 
(1)
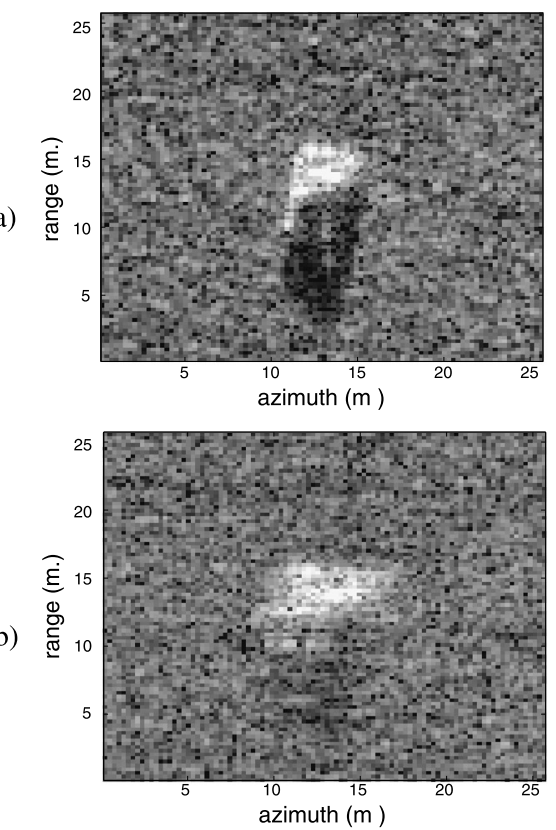

(c)

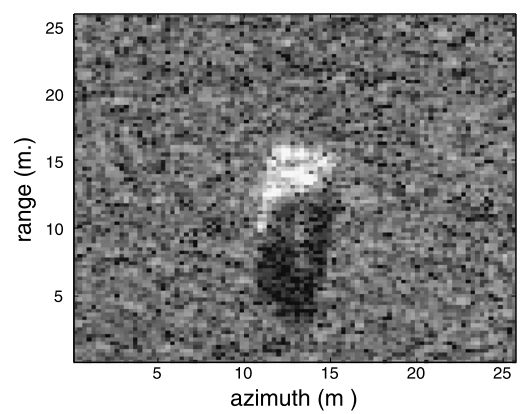

(d)

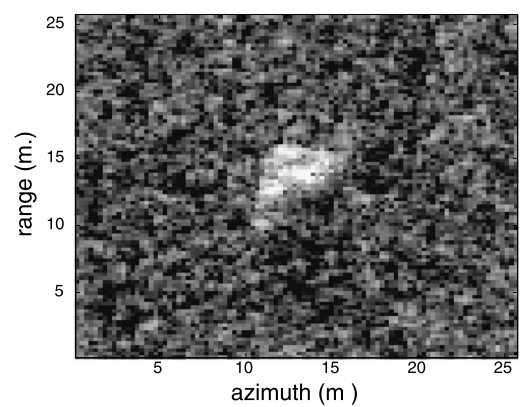

(e)

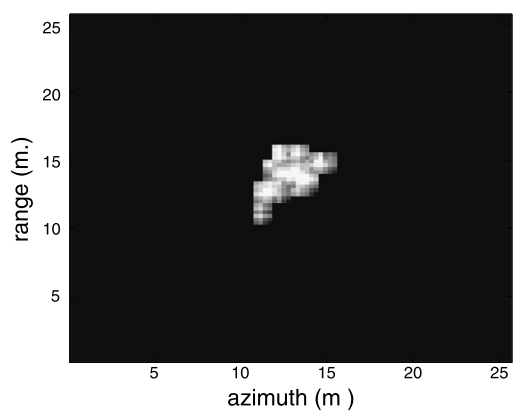

(2)
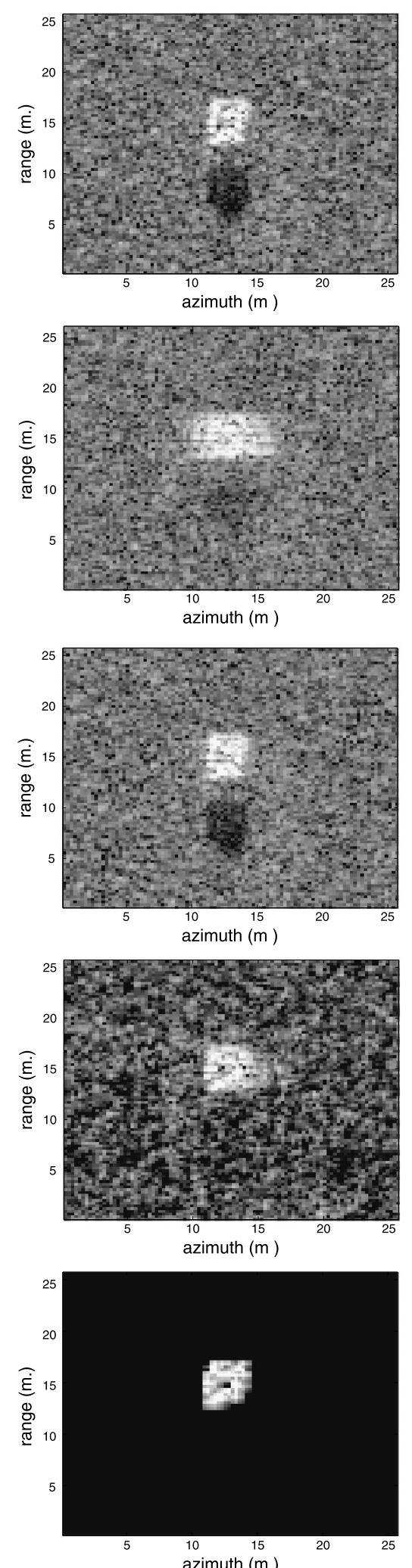

(3)
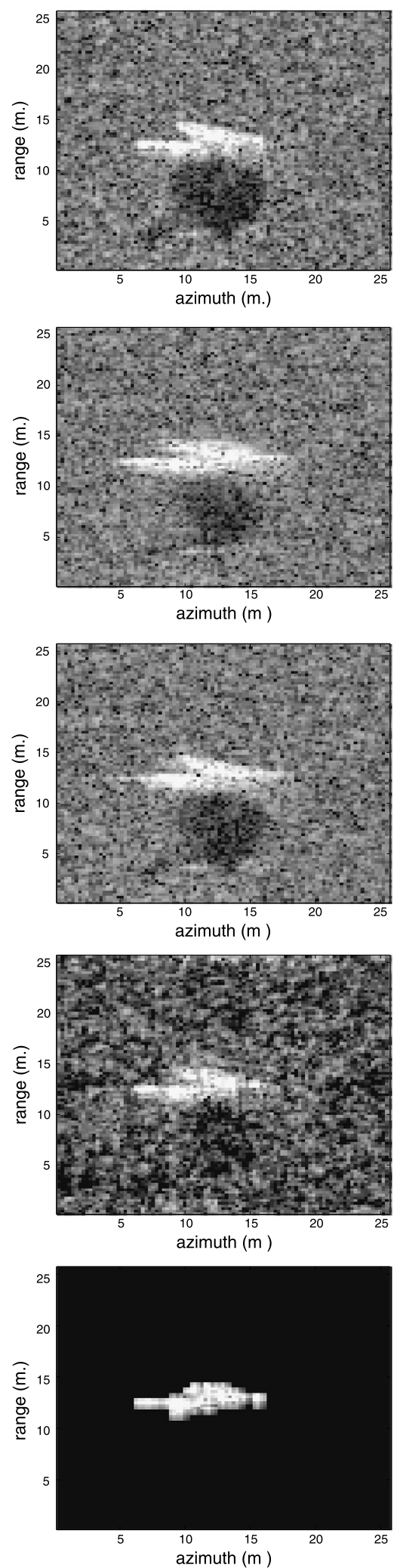

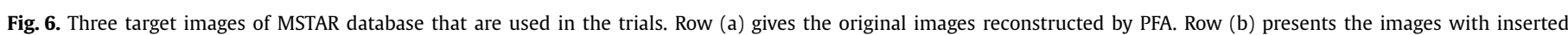

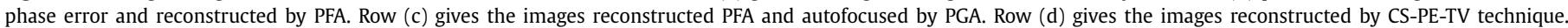
Row (e) shows the images reconstructed and autofocused by the proposed EMMP-AF-SAR technique. 
Table 4

Comparison of performance metrics for the imagery reconstructed by techniques PFA-PGA, CS-PE-TV, and EMMP-AF-SAR.

\begin{tabular}{llll}
\hline & & MSE & Entropy \\
\hline Target 1 & PFA-PGA & $\mathbf{2 . 5} \times \mathbf{1 0}$ & $2 . \mathbf{4}$ \\
& CS-PE-TV & $3.6 \times 10^{-3}$ & 2.42 \\
Target 2 & EMMP-AF-SAR & $1.9 \times 10^{-3}$ & $\mathbf{0 . 3 2}$ \\
& PFA-PGA & $\mathbf{1 . 9} \times \mathbf{1 0}^{-\mathbf{3}}$ & 2.95 \\
& CS-PE-TV & $4.5 \times 10^{-3}$ & 2.94 \\
Target 3 & EMMP-AF-SAR & $5.4 \times 10^{-3}$ & $\mathbf{0 . 3 3}$ \\
& PFA-PGA & $\mathbf{9 . 3} \times \mathbf{1 0}^{-\mathbf{4}}$ & 2.80 \\
& CS-PE-TV & $7.2 \times 10^{-3}$ & 2.42 \\
& EMMP-AF-SAR & $9.6 \times 10^{-3}$ & $\mathbf{0 . 4 1}$ \\
\hline
\end{tabular}

\section{Conclusions}

SAR imaging of scenes with strong man-made targets is of interest in many remote sensing applications. The required high resolution in these images can only be obtained with an effective compensation of errors induced by platform motion.

In this work, a new EMMP algorithm based SAR image reconstruction technique is proposed that provides accurate estimation of phase errors due to uncompensated platform motion and delivers high quality reconstructions of sparse target scenes. It is demonstrated on both synthetic and real SAR data that the proposed EMMP based autofocused SAR reconstruction technique provides efficient reconstructions of SAR images even under severe phase errors. Since, it requires only a fraction of the Nyquist rate samples, the EMMP-AF-SAR technique also relaxes the requirements on the SAR hardware.

Unlike alternative $l_{1}$ norm minimization based approaches, the proposed technique provides near optimal solution to the desired $l_{0}$ norm minimization problem efficiently by a sequential search procedure in 1-dimensional search spaces. Moreover, EMMP-AFSAR technique converges faster compared to Non-Linear Conjugate Gradient Descent algorithm for CS reconstructions while providing comparable quality outputs. An example image reconstruction with the non-linear conjugate gradient descent algorithm takes about 20 hours due to large matrix operations with high computational complexities. However the same reconstruction with the EMMP-AF-SAR technique takes only 3-5 minutes thanks to its lower computational complexity. The 3-5 minutes reconstruction time is slightly longer than the reconstruction time of the PFA-PGA technique which is around one minute. Comparison with PFA-PGA technique over synthetic and real data sets with manmade targets shows that the proposed technique provides comparable or improved reconstructions. In addition, since EMMP-AF-SAR reconstructions are highly localized with significantly suppressed speckle, it enables improved target classification.

\section{Appendix A. Supplementary material}

Supplementary material related to this article can be found online at http://dx.doi.org/10.1016/j.dsp.2014.11.001.

\section{References}

[1] C.E. Mancill, J.M. Swiger, A mapdrift autofocus technique for correcting higher order SAR phase errors, in: 27th Annual Tri-Service Radar Symposium Record, 1981, pp. 391-400

[2] L. Xi, L. Guosui, J. Ni, Autofocusing of ISAR images based on entropy minimization, IEEE Trans. Aerosp. Electron. Syst. 35 (4) (1999) 1240-1252.

[3] C.V. Jakowatz Jr., D.E. Wahl, Eigenvector method for maximum-likelihood estimation of phase errors in synthetic-aperture-radar imagery, J. Opt. Soc. Am. A 10 (12) (1993) 2539-2546.

[4] R.L. Morrison, M.N. Do, D.C. Munson Jr., SAR image autofocus by sharpness optimization: a theoretical study, IEEE Trans. Image Process. 16 (9) (2007) 2309-2321.
[5] R.L. Morrison, M.N. Do, D.C. Munson Jr., MCA: a multichannel approach to SAR autofocus, IEEE Trans. Image Process. 18 (4) (2009) 840-853.

[6] P.H. Eichel, D.C. Ghiglia, C.V. Jakowatz, Speckle processing method for synthetic aperture radar phase correction, Opt. Lett. 14 (1989) 1-3.

[7] D.E. Wahl, P.H. Eichel, D.C. Ghiglia, C.V. Jakowatz, Phase gradient autofocus - a robust tool for high resolution SAR phase correction, IEEE Trans. Aerosp. Electron. Syst. 30 (1994) 827-835.

[8] D.L. Donoho, Compressed sensing, IEEE Trans. Inf. Theory 52 (4) (2006) 1289-1306.

[9] E. Candès, T. Tao, Near-optimal signal recovery from random projections: universal encoding strategies?, IEEE Trans. Inf. Theory 52 (12) (2006) 5406-5425.

[10] D.L. Donoho, M. Elad, Optimally sparse representation in general (nonorthogonal) dictionaries via 11 minimization, Proc. Natl. Acad. Sci. 100 (2003) 2197-2202

[11] E. Candès, M.B. Wakin, An introduction to compressive sampling, IEEE Signal Process. Mag. 25 (2) (2008) 21-30.

[12] L.C. Potter, E. Ertin, J.T. Parker, M. Cetin, Sparsity and compressed sensing in radar imaging, Proc. IEEE 98 (6) (2010) 1006-1020.

[13] M. Herman, T. Strohmer, Compressed sensing radar, in: IEEE Radar Conference, 2008, pp. 1-6.

[14] M. Herman, T. Strohmer, High resolution radar via compressed sensing, IEEE Trans. Signal Process. 57 (6) (2009) 2275-2284.

[15] G.E. Smith, T. Diethe, Z. Hussain, J.S. Taylor, D.R. Hardoon, Compressed sampling for pulse Doppler radar, in: IEEE Radar Conference, 2010, pp. 887-892.

[16] J.H.G. Ender, On compressive sensing applied to radar, Signal Process. 90 (5) (2010) 1402-1410.

[17] M. Weiss, Target detection with a distributed WLAN radar network using compressive sampling, in: 1st International Workshop on Compressed Sensing Applied to Radar, 2012.

[18] A.C. Gürbüz, J.H. McClellan, W.R. Scott, A compressive sensing data acquisition and imaging method for stepped frequency GPRs, IEEE Trans. Signal Process. 57 (7) (2009) 2640-2650.

[19] R. Baraniuk, P. Steeghs, Compressive radar imaging, in: IEEE Radar Conference, 2007, pp. 128-133.

[20] S. Uğur, O. Arıkan, SAR image reconstruction and autofocus by compressed sensing, Digit. Signal Process. 22 (6) (2012) 923-932.

[21] G. Rilling, M. Davies, B. Mulgrew, Compressed sensing based compression of SAR raw data, in: SPARS'09 - Signal Processing with Adaptive Sparse Structured Representations, 2009.

[22] S. Bhattacharya, T. Blumensath, B. Mulgrew, M. Davies, Fast encoding of synthetic aperture radar raw data using compressed sensing, in: IEEE/SP 14th Workshop on Statistical Signal Processing, 2007, pp. 448-452.

[23] M. Cetin, W.C. Karl, Feature-enhanced synthetic aperture radar image formation based on nonquadratic regularization, IEEE Trans. Image Process. 10 (4) (2001) 623-631.

[24] S. Samadi, M. Cetin, M.A. Masnadi-Shirazi, Sparse representation based synthetic aperture radar imaging, IET Radar Sonar Navig. 5 (2) (2011) 182-193.

[25] V.M. Patel, G.R. Glenn, D.M. Healy, R. Chellappa, Compressed synthetic aperture radar, IEEE J. Sel. Top. Signal Process. 4 (2) (2010) 244-254.

[26] M. Cetin, I. Stojanović, N.O. Önhon, K.R. Varshney, S. Samadi, W.C. Karl, A.S. Willsky, Sparsity-driven synthetic aperture radar imaging, IEEE Signal Process. Mag. 31 (4) (2014) 27-40

[27] S.I. Kelly, G. Rilling, M. Davies, B. Mulgrew, Iterative image formation using fast (re/back)-projection for spotlight-mode SAR, in: IEEE Radar Conference, 2011, pp. 835-840.

[28] N.O. Önhon, M. Cetin, Joint sparsity-driven inversion and model error correction for radar imaging, in: IEEE International Conference on Acoustics Speech and Signal Processing, 2010, pp. 1206-1209.

[29] N.O. Önhon, M. Cetin, A sparsity driven approach for joint SAR imaging and phase error correction, IEEE Trans. Image Process. 21 (4) (2012) 2075-2088.

[30] A.C. Gürbüz, M. Pilancı, O. Arıkan, Expectation maximization based matching pursuit, in: IEEE International Conference on Acoustics Speech and Signal Processing, 2012.

[31] S. Uḡur, A.C. Gürbüz, O. Arıkan, SAR image reconstruction by EMMP algorithm, in: IEEE 20th Conference on Signal Processing and Communication Applications, Antalya, Turkey, 2012

[32] S. Uḡur, A.C. Gürbüz, O. Arıkan, Autofocused sparse SAR image reconstruction by EMMP algorithm, in: 1st International Workshop on Compressed Sensing Applied to Radar, Bonn, Germany, 2012.

[33] R. Baraniuk, Compressive sensing, IEEE Signal Process. Mag. 24 (4) (2007) $118-121$

[34] E. Candès, J. Romberg, T. Tao, Stable signal recovery from incomplete and inaccurate measurements, Commun. Pure Appl. Math. 59 (8) (2006) 1207-1223.

[35] E. Candès, J. Romberg, T. Tao, Robust uncertainty principles: exact signal reconstruction from highly incomplete frequency information, IEEE Trans. Inf. Theory 52 (2) (2006) 489-509.

[36] E. van den Berg, M.P. Friedlander, Probing the Pareto frontier for basis pursuit solutions, SIAM J. Sci. Comput. 31 (2) (2009) 890-912.

[37] R. Tibshirani, Regression shrinkage and selection via the Lasso, J. R. Stat. Soc. B 58 (1996) 267-288 
[38] C.V. Jakowatz Jr., D.E. Wahl, P.H. Eichel, D.C. Ghiglia, P.A. Thompson, SpotlightMode Synthetic Aperture Radar: A Signal Processing Approach, Springer Science+Business Media, LLC, 1996.

[39] W. Boyd, L. Vandenberghe, Convex Optimization, Cambridge University Press, 2004.

[40] A. Dempster, N. Laird, D. Rubin, Maximum likelihood from incomplete data via the EM algorithm, J. R. Stat. Soc. B 39 (1) (1977) 1-38.

[41] https://www.sdms.afrl.af.mil, the Sensor Data Management System MSTAR public data.

[42] H.J. Cho, D.C. Munson Jr., Multichannel SAR autofocus using multiple lowreturn constraints, in: IEEE International Conference on Acoustics Speech and Signal Processing, 2010, pp. 1346-1349.

[43] K. Köse, V. Cevher, A.E. Cetin, Filtered variation method for denoising and sparse signal processing, in: IEEE International Conference on Acoustics Speech and Signal Processing, 2012, pp. 3329-3332.

Salih Uğur was born in 1971 in Ankara, Turkey. He received the B.Sc. degree in Electrical and Electronics Engineering from Middle East Technical University, Ankara, Turkey, in 1993. He received both his M.S. and Ph.D. degrees in Electrical and Electronics Engineering from Bilkent University, Ankara, Turkey, in 1996 and 2013, respectively. He currently works for Meteksan Savunma, Ankara, Turkey on radar signal processing. His research interests include radar signal processing, remote sensing and compressed sensing.

Orhan Arıkan was born in 1964 in Manisa, Turkey. In 1986, he received his B.Sc. degree in Electrical and Electronics Engineering from the
Middle East Technical University, Ankara, Turkey. He received both his M.S. and Ph.D. degrees in Electrical and Computer Engineering from the University of Illinois, Urbana-Champaign, in 1988 and 1990, respectively. Following his graduate studies, he was employed as a Research Scientist at Schlumberger-Doll Research Center, Ridgefield, CT. In 1993 he joined the Electrical and Electronics Engineering Department of Bilkent University, Ankara, Turkey. Since 2006, he is a full professor at Bilkent University. His current research interests include statistical signal processing, timefrequency analysis and remote sensing. Dr. Arikan has served as chairman of Turkey chapter of IEEE Signal Processing Society and president of IEEE Turkey Section. Since 2011, he is serving as the chairman of the Department of Electrical and Electronics Engineering.

Ali Cafer Gürbüz received the B.S. degree from Bilkent University, Ankara, Turkey, in 2003 in Electrical and Electronics Engineering, and the M.S. and Ph.D. degrees from the Georgia Institute of Technology, Atlanta, in 2005 and 2008, both in Electrical and Computer Engineering, respectively. From 2003 to 2008, he participated in multimodal landmine detection system research as a Graduate Research Assistant and from 2008 to 2009, as Postdoctoral Fellow, all with Georgia Tech. He is currently an Assistant Professor with TOBB University of Economics and Technology, Ankara, Turkey with the Department of Electrical and Electronics Engineering. His research interests include compressive sensing applications, ground penetrating radar, array signal processing, remote sensing and imaging. 